\title{
Phase II study of capecitabine plus oxaliplatin (CapOX) as adjuvant chemotherapy for locally advanced rectal cancer (CORONA II)
}

\author{
Norifumi Hattori ${ }^{1}\left[\right.$ ( Goro Nakayama ${ }^{1} \cdot$ Keisuke Uehara ${ }^{2} \cdot$ Toshisada Aiba $^{2} \cdot$ Kiyoshi Ishigure $^{3} \cdot$ Eiji Sakamoto $^{4}$. \\ Yuichiro Tojima ${ }^{5} \cdot$ Mitsuro Kanda $^{1}$ - Daisuke Kobayashi ${ }^{1}$. Chie Tanaka ${ }^{1}$. Suguru Yamada ${ }^{1} \cdot$ Masahiko Koike $^{1}$. \\ Michitaka Fujiwara ${ }^{1} \cdot$ Masato Nagino ${ }^{2} \cdot$ Yasuhiro Kodera ${ }^{1}$
}

Received: 15 March 2019 / Accepted: 10 September 2019 / Published online: 21 September 2019

(c) The Author(s) 2019

\begin{abstract}
Objective This multicenter, single-arm phase II study (UMIN000008429) aimed to evaluate the efficacy and safety of capecitabine plus oxaliplatin (CapOX) as postoperative adjuvant chemotherapy for patients with locally advanced rectal cancer. Methods Patients with resectable clinical Stage II or III rectal cancer were enrolled to receive eight cycles of CapOX therapy $\left(130 \mathrm{mg} / \mathrm{m}^{2}\right.$ oxaliplatin on day 1 and $2000 \mathrm{mg} / \mathrm{m}^{2}$ oral capecitabine on days $1-14$, every 3 weeks) after curative surgical resection. The primary endpoint was 3-year relapse-free survival (RFS) rate, and secondary endpoints were 3-year overall survival (OS) rate, treatment compliance, and safety.

Results A total of 40 patients (Stage II, 21; Stage III, 19) were enrolled between September 2012 and November 2015 from seven institutions. Thirty-nine patients (97\%) received R0 resection, and 32 patients (84\%) received postoperative CapOX therapy. The completion rate of all eight cycles of CapOX therapy was $66 \%$. Relative dose intensities were $87 \%$ for oxaliplatin and $84 \%$ for capecitabine. At a median follow-up period of 46 months, disease recurrence was observed in nine patients, including three with local recurrence. Three-year RFS and OS rates were 75\% (95\% CI 57-86\%) and 96\% (95\% CI 80-99\%), respectively. Frequencies of Grade $\geq 3$ hematological and non-hematologic adverse events were $19 \%$ and 38\%, respectively. Conclusion CapOX therapy is feasible as adjuvant chemotherapy for locally advanced rectal cancer.
\end{abstract}

\section{Introduction}

Colorectal cancer is one of the leading causes of cancer death, and rectal cancer represents about $40 \%$ of all colorectal cancers [1]. The standard treatment for locally limited disease has been resection of the primary lesion with adequate lymphadenectomy. In Japan, a combination of surgery including lateral lymph node dissection and postoperative

Norifumi Hattori

norifumi@med.nagoya-u.ac.jp

1 Department of Gastroenterological Surgery, Nagoya University Graduate School of Medicine, 65 Tsurumai-cho, Showa-ku, Nagoya, Aichi 466-8550, Japan

2 Department of Surgical Oncology, Nagoya University Graduate School of Medicine, Nagoya, Japan

3 Department of Surgery, Konan Kosei Hospital, Konan, Japan

4 Department of Surgery, Nagoya Daini Red Cross Hospital, Nagoya, Japan

5 Department of Surgery, Chukyo Hospital, Nagoya, Japan adjuvant chemotherapy represents the standard treatment of resectable locally advanced rectal cancer [2]. On the other hand, preoperative CRT for advanced lower rectal cancer is commonly performed in Europe and the United States [3-6], and few reports exist on the outcomes of treatment comprising postoperative adjuvant chemotherapy alone. For colon cancer, surgical resection followed by adjuvant chemotherapy with fluoropyrimidine has been established as the standard treatment for locally advanced disease [7-13]. Furthermore, several randomized controlled trials have shown that adjuvant chemotherapy with oxaliplatin in combination with fluoropyrimidine improves the survival of patients with resected Stage III colon cancer [9-11].

Clinical evidence on the efficacy of adjuvant chemotherapy for rectal cancer is relatively limited compared with those for colon cancer. The NSAS-CC trial showed that adjuvant chemotherapy with UFT, an oral fluoropyrimidine, following curative resection for Stage III rectal cancer had a survival benefit compared with surgery alone [14]. The ACTS-RC trial, which compared UFT with S-1 for Stage II/III rectal cancer, showed that S-1 therapy was superior 
to UFT with respect to recurrence-free survival time [15]. However, the efficacy and safety of oxaliplatin-based adjuvant chemotherapy for locally advanced rectal cancer remain unclear.

Thus, this multicenter, single-arm phase II study aimed to examine the efficacy and safety of capecitabine plus oxaliplatin (CapOX) as postoperative adjuvant chemotherapy in Japanese patients with locally advanced rectal cancer.

\section{Patients and methods}

\section{Study design}

This multicenter, single-arm phase II clinical trial was conducted by the Chubu Clinical Oncology Group (CCOG) and Nagoya Society of Oncology Group (NSOG) at seven hospitals in Japan. Inclusion criteria were as follows: age $\geq 20$ years, histologically proven rectal adenocarcinoma located below the peritoneal reflection, locally advanced rectal cancer with clinical T3-4 tumor or positive lymph nodes, lack of distant metastasis, no prior chemotherapy and radiotherapy, Eastern Cooperative Oncology Group (ECOG) performance status of 0 or 1 , and adequate hematologic, hepatic, and renal function. Patients with clinically significant cardiovascular disease, double cancer, bowel obstruction, Grade $\geq 1$ peripheral sensory neuropathy (PSN), or uncontrolled diabetes mellitus were excluded.

This study was conducted in accordance with the principles set forth in the Declaration of Helsinki. Patients provided written informed consent prior to participation. The ethics committees of Nagoya University Hospital (approval number 2014-0043) and all other participating facilities approved the study. This trial was registered with the University Hospital Medical Information Network (UMIN000008429).

\section{Treatment plan}

Patients were enrolled within 2 weeks before surgery, which included total mesorectal excision (TME) or tumor-specific mesorectal excision (TSME) with adequate lymphadenectomy according to Japanese guidelines for the treatment of colorectal cancer. For patients who required extended resection to achieve $R 0$ curative resection, combined resection of the autonomic nerve, pelvic vessels, and adjacent organs was performed at the surgeons' discretion. As adjuvant chemotherapy, CapOX therapy, which consisted of intravenous infusion of $130 \mathrm{mg} / \mathrm{m}^{2}$ oxaliplatin on day 1 , along with oral administration of $2000 \mathrm{mg} / \mathrm{m}^{2}$ capecitabine on days $1-14$, repeated every 3 weeks, was initiated in patients with pathological T3-4 tumors or positive lymph nodes within 8 weeks after surgery. Treatment was continued until completion of eight cycles or any one of the following occurred: disease progression, unacceptable toxicity, deterioration of ECOG performance status to $>2$, or withdrawal of patient consent. Dose modification in response to treatment-related toxicities was carried out in accordance with the study protocol.

\section{Endpoints}

The primary endpoint was 3-year relapse-free survival (RFS) rate, with RFS defined as the time from surgery until disease recurrence or death from any cause. Secondary endpoints included 3-year overall survival (OS) rate, with OS defined as the time from surgery until death from any cause; cumulative local and distant recurrence rates; $R 0$ resection rate; treatment compliance; and adverse events. Recurrence was assessed by computed tomography every 6 months after surgery and total colonoscopy once every year. Adverse events were graded according to the National Cancer Institute Common Terminology Criteria (NCI-CTC), version 3.0.

All analyses of efficacy were based on the intent-to-treat (ITT) population, defined as eligible and assessable enrolled patients. The safety population was defined as all patients receiving $\geq 1$ dose of the protocol treatment.

\section{Statistical analysis}

Statistical power was calculated based on the following assumptions: 3-year RFS rate threshold of 50\% and an expected 3-year RFS rate of $70 \%$ based on data from previous clinical trials [14], with an enrollment period of 2 years and follow-up period of 5 years. To ensure an alpha level of 0.05 (one-side) and a detection power $(1-\beta)$ of $80 \%, 37$ patients were required. Therefore, the planned sample size was 40 patients to account for possible loss to follow-up. Differences in characteristics between the two arms were analyzed using the $\chi^{2}$ test for categorical variables and the Mann-Whitney $U$ test for continuous variables. Time-to-event variables, RFS, and OS were analyzed by the Kaplan-Meier method. $P$ values less than 0.05 were considered statistically significant. Statistical analyses were performed using JMP software version 13 (SAS Institute Inc., Cary, NC, USA).

\section{Results}

\section{Patient characteristics}

A total of 40 patients were prospectively enrolled between September 2012 and November 2015 from 7 institutions. The ITT population comprised 40 patients. Eight patients were excluded from the safety analysis for the following reasons: two were diagnosed with pathological Stage I disease: 


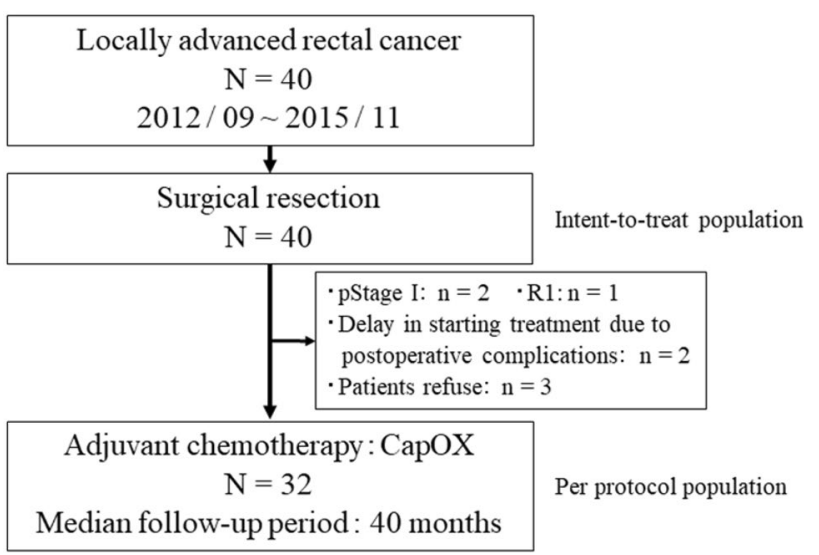

Fig. 1 Consort diagram. A total of 40 patients enrolled between September 2012 and November 2015 underwent surgical resection. CapOX adjuvant chemotherapy was administered to 32 patients. $N$ total number of patients, $n$ number of patients, CapOX capecitabine and oxaliplatin

(1) received non-curative resection, (2) had postoperative complications, and (3) refused protocol treatment. Thus, the safety population comprised 32 patients (Fig. 1). Baseline clinical characteristics are summarized in Table 1. The relationship between clinical and pathological staging is shown in Table 2. The agreement between clinical and pathological TN factors was $60 \%$ (24/40) and 67.5\% (27/40), respectively. The correlation between cStage and pStage resulted in an agreement of $75 \%$ (30/40).

\section{Surgical procedures}

All 40 patients received surgical procedures, including low anterior resection with TME in 20 patients and with TSME in nine patients, and Miles' operation in nine patients. $R 0$ resection was achieved in 39 patients (98\%). The median number of dissected lymph nodes was 22 (range 6-47). Postoperative complications (Clavien-Dindo classification Grade 2 and higher) were observed in 13 patients (33\%), including surgical site infection in four, ileus in six, anastomotic leakage in three, and dysuria in three.

\section{Treatment exposure}

Thirty-two patients $(82 \%)$ were treated with CapOX therapy. The median time from surgery to initiation of adjuvant chemotherapy was 43 days (range 10-56 days). The median number of cycles was eight (range 1-8 cycles), and the overall treatment completion rate was $66 \%$. The relative dose intensity was $87 \%$ (range 12-100\%) for oxaliplatin and $84 \%$ (range $12.5-100 \%$ ) for capecitabine (Table 3).
Table 1 Patient characteristics

\begin{tabular}{|c|c|c|c|}
\hline \multirow[t]{2}{*}{ Variable } & \multicolumn{3}{|c|}{$N=40$} \\
\hline & $n$ & & $\%$ \\
\hline \multicolumn{4}{|l|}{ Gender } \\
\hline Male & 25 & & 63 \\
\hline Female & 15 & & 37 \\
\hline \multicolumn{4}{|l|}{ Age years } \\
\hline Median (range) & & $68(49-80)$ & \\
\hline \multicolumn{4}{|l|}{ PS (ECOG) } \\
\hline 0 & 35 & & 88 \\
\hline 1 & 5 & & 12 \\
\hline \multicolumn{4}{|l|}{ BMI } \\
\hline Median (range) & & $21.5(13.5-31.4)$ & \\
\hline \multicolumn{4}{|l|}{ Tumor diameter (mm) } \\
\hline Median (range) & & $40(20-80)$ & \\
\hline \multicolumn{4}{|c|}{ Distance from anal verge to tumor $(\mathrm{cm})$} \\
\hline Median (range) & & $5.5(0.5-15)$ & \\
\hline \multicolumn{4}{|l|}{$\mathrm{cT}$} \\
\hline $\mathrm{T} 2$ & 4 & & 10 \\
\hline $\mathrm{T} 3$ & 24 & & 60 \\
\hline $\mathrm{T} 4$ & 12 & & 30 \\
\hline \multicolumn{4}{|l|}{$\mathrm{cN}$} \\
\hline NO & 21 & & 53 \\
\hline$N 1$ & 14 & & 35 \\
\hline$N 2$ & 5 & & 12 \\
\hline \multicolumn{4}{|l|}{ cStage } \\
\hline II & 21 & & 53 \\
\hline III & 19 & & 47 \\
\hline \multicolumn{4}{|l|}{ Pathology } \\
\hline Tub & 38 & & 95 \\
\hline por/muc & 2 & & 5 \\
\hline \multicolumn{4}{|l|}{ Operative procedure } \\
\hline Low anterior resection & 29 & & 73 \\
\hline Abdominal peritoneal resection & 9 & & 23 \\
\hline Hartmann operation & 1 & & 2 \\
\hline Intersphincteric resection & 1 & & 2 \\
\hline \multicolumn{4}{|l|}{ Approach } \\
\hline Open & 13 & & 33 \\
\hline Laparoscopy & 27 & & 67 \\
\hline \multicolumn{4}{|l|}{ Lateral lymph node dissection } \\
\hline Bilateral & 7 & & 18 \\
\hline Unilateral & 2 & & 4 \\
\hline None & 31 & & 78 \\
\hline
\end{tabular}

$N$ total number of patients, $n$ number of patients, $P S$ performance status, ECOG Eastern Cooperative Oncology Group, BMI body mass index

\section{Efficacy}

Data cutoff for the final analysis was set as December 1, 2018. The median length of follow-up for censored cases 
Table 2 Relationship between clinical and pathological staging

\begin{tabular}{|c|c|c|c|c|}
\hline & $\mathrm{pTl}$ & pT2 & pT3 & pT4 \\
\hline $\mathrm{cT} 2(n=4)$ & 1 & 1 & 2 & \\
\hline cT3 $(n=24)$ & & 2 & 19 & 3 \\
\hline \multirow[t]{2}{*}{ cT4 $(n=12)$} & & 1 & 7 & 4 \\
\hline & pNO & $\mathrm{pNl}$ & $\mathrm{pN} 2$ & $\mathrm{pN} 3$ \\
\hline $\mathrm{cN0}(n=21)$ & 17 & 3 & 1 & \\
\hline $\mathrm{cNl}(n=14)$ & 4 & 7 & 3 & \\
\hline $\mathrm{cN} 2(n=5)$ & & 1 & 3 & 1 \\
\hline \multicolumn{5}{|l|}{$\mathrm{cN} 3(n=0)$} \\
\hline & \multicolumn{2}{|c|}{ pStage I } & pStage II & pStage III \\
\hline cStage II $(n=21)$ & \multirow[t]{2}{*}{2} & \multicolumn{2}{|r|}{15} & 4 \\
\hline cStage III $(n=19)$ & & & 4 & 15 \\
\hline
\end{tabular}

$n$ number of patients

Table 3 Adjuvant treatment status

\begin{tabular}{ll}
\hline & $N=32$ \\
\hline $\begin{array}{l}\text { Time from surgery to initiation of chemotherapy } \\
\text { (days) }\end{array}$ & \\
Median (range) & $43(10-56)$ \\
Treatment cycles received & \\
Median (range) & $8(1-8)$ \\
Completion rate (\%) & $66 \%$ \\
RDI (\%) & \\
Capecitabine & \\
Median (range) & $84(12.5-100)$ \\
Oxaliplatin & $87(12-100)$ \\
Median (range) &
\end{tabular}

$N$ total number of patients, $R D I$ relative dose intensity

Table 4 Recurrence pattern

\begin{tabular}{lll}
\hline & $N=32$ \\
\cline { 2 - 3 } & $n \quad \%$ \\
\hline Recurrence & 9 & 28 \\
Local recurrence & 3 & 9 \\
Anastomotic & 1 & \\
Pelvic & 2 & \\
Distant recurrence & 8 & 25 \\
Liver & 1 \\
Lung & 2 \\
Para-aortic lymph node & 5 \\
\hline$N$ total number of patients, $n$ \\
number of patients
\end{tabular}

was 46 months (range 25-62 months). Disease recurrence after adjuvant chemotherapy was observed in nine patients (28\%), including three with local recurrence and eight
A Relapse-free survival (ITT population)

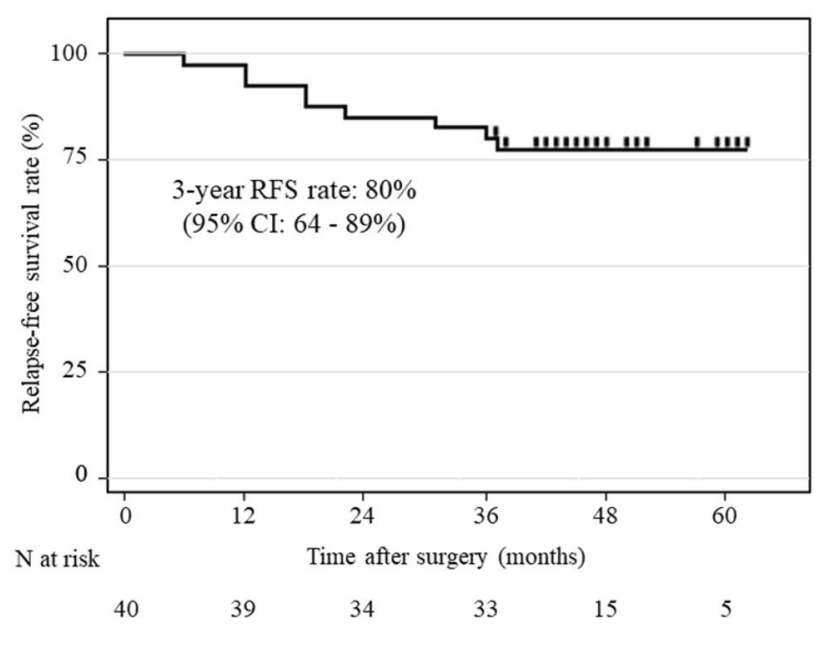

B Overall survival (ITT population)

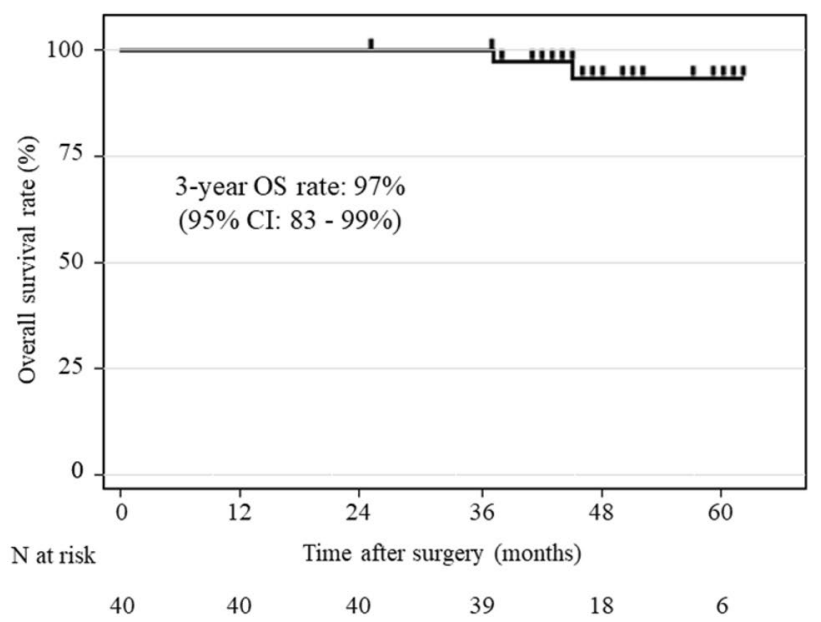

Fig. 2 Survival outcomes (ITT population). a Three-year RFS rate was $80 \%$ (95\% CI 64-89\%). b Three-year OS rate was $97 \%$ (95\% CI $83-99 \%)$. RFS relapse-free survival, $O S$ overall survival, $N$ total number of patients

with distant recurrence (Table 4). The 3-year RFS rate (primary endpoint) was 80\% (95\% CI 64-89\%) (Fig. 2a). The 3-year OS rate was 97\% (95\% CI 83-99\%) (Fig. 2b). In the per protocol population, 3-year RFS and OS rates were $75 \%$ (95\% CI 57-86\%) and 96\% (95\% CI 80-99\%), respectively (Fig. 3a, b). In the prognosis of each staging, the 3-year RFS rate was 93\% (95\% CI 64-99\%) in pStage II and 52\% (95\% CI 30-74\%) in pStage III, and the 3-year OS rate was $100 \%$ in pStage II and 93\% (95\% CI 66-99\%) in pStage III. Three-year cumulative rates for local recurrence and distant recurrence were 9.3\% (95\% CI 3.0-25\%) and $21 \%$ (95\% CI 10-39\%), respectively (Fig. 3c). 
Fig. 3 Survival outcomes (per protocol population). a Three-year RFS rate was $75 \%$ (95\% CI 57-86\%). b Three-year OS rate was $96 \%$ (95\% CI 80-99\%). c Three-year cumulative rate of local recurrence and distant recurrence were $9.3 \%$ (95\% CI 3.0-25\%) and $21 \%(95 \%$ CI $10-39 \%$ ), respectively. RFS relapse-free survival, OS overall survival, $N$ total number of patients

\section{Safety}

The incidence of treatment-related adverse events is shown in Table 5. Frequencies of Grade $\geq 3$ hematologic and nonhematologic adverse events were $19 \%$ and $38 \%$, respectively. The most frequent Grade $\geq 3$ hematologic and non-hematologic adverse events were thrombocytopenia (12.5\%) and PSN (15.6\%), respectively. The incidence of toxicity-related treatment discontinuation was $73 \%$, and the most common adverse event that led to discontinuation was PSN (38\%). PSN lasting after the end of adjuvant treatment was experienced by eight patients. No patient had persistent Grade $\geq 3$ PSN.

\section{Discussion}

This phase II trial was conducted to evaluate the efficacy and safety of CapOX as postoperative adjuvant chemotherapy for Stage II/III rectal cancer. The 3-year RFS rate (primary endpoint) of $80 \%$ was considered acceptable according to our hypothesis that assumes threshold and expected survival rates of $50 \%$ and $70 \%$, respectively. Furthermore, the 3-year RFS and OS rate (per protocol population) were $75 \%$ and $96 \%$, comparable to those reported in two RCTs evaluating adjuvant chemotherapy for resectable rectal cancer without preoperative therapy $[14,15]$.

Multimodal therapy comprising preoperative fluoropyrimidine with concurrent radiotherapy followed by TME and adjuvant fluoropyrimidine-based chemotherapy is recommended as a global standard for patients with Stage II/III rectal cancer [16, 17]. The reported 3-year RFS and local recurrence rates of neoadjuvant CRT plus adjuvant chemotherapy for patients with locally advanced rectal cancer range from 62.9 to $72.7 \%$ and 4.4 to $12.1 \%$, respectively [18-20]. Although we could not directly compare prognosis between patients who received postoperative adjuvant chemotherapy alone and neoadjuvant CRT plus adjuvant chemotherapy, the 3-year RFS and local recurrence rates in our study were comparable to treatment outcomes for neoadjuvant CRT plus adjuvant chemotherapy. Surprisingly, these favorable outcomes were achieved by adjuvant chemotherapy alone following curative resection.

The benefits of oxaliplatin-based neoadjuvant CRT and adjuvant chemotherapy in Stage II/III rectal cancer are controversial. At least seven randomized trials [18-24] have
A Relapse-free survival (Per protocol population)

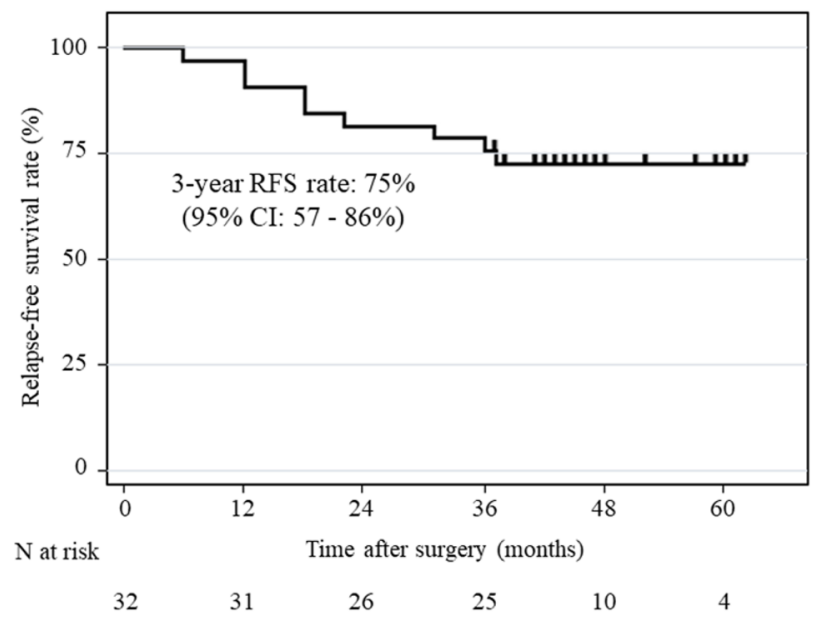

B Overall survival (Per protocol population)

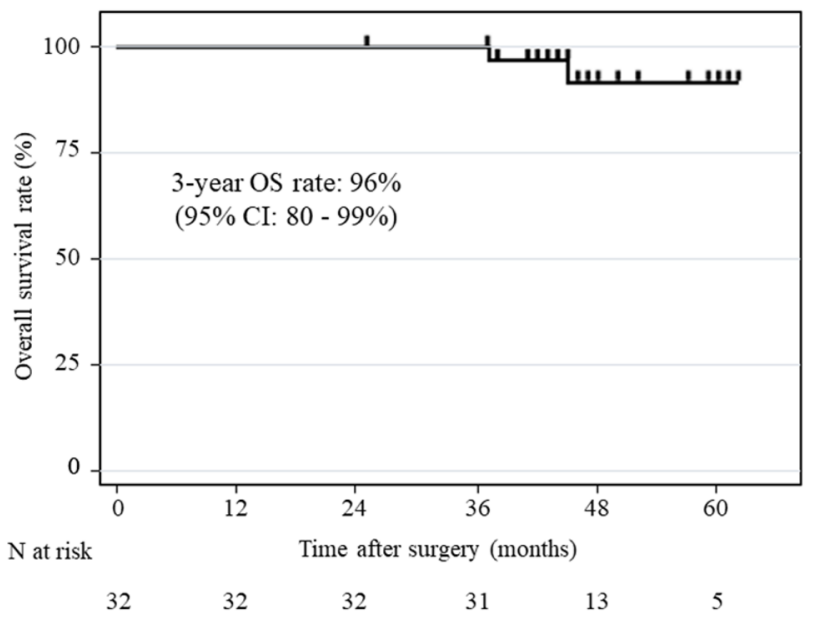

C Cumulative recurrence rate (Per protocol population)

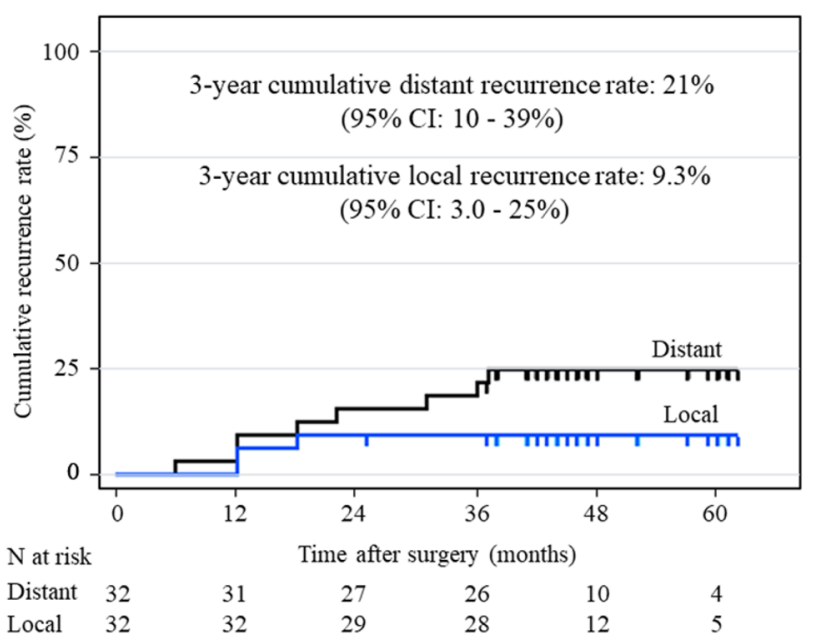


Table 5 Frequency of common toxicities

\begin{tabular}{|c|c|c|c|c|}
\hline & \multicolumn{4}{|c|}{$N=32$} \\
\hline & \multicolumn{2}{|c|}{ All grade } & \multicolumn{2}{|c|}{$\geq$ Grade 3} \\
\hline & $n$ & $\%$ & $n$ & $\%$ \\
\hline Hematologic toxicity & 21 & 65.6 & 6 & 19 \\
\hline Neutropenia & 17 & 53.1 & 3 & 9.3 \\
\hline Anemia & 21 & 65.6 & 1 & 3.1 \\
\hline Thrombocytopema & 21 & 65.6 & 4 & 12.5 \\
\hline Febrile neutropenia & 0 & 0 & 0 & 0 \\
\hline Non-hematologic toxicity & 26 & 81.2 & 12 & 38 \\
\hline Anorexia & 18 & 56.2 & 4 & 12.5 \\
\hline Diarrhea & 8 & 25 & 1 & 3.1 \\
\hline Nausea/vomiting & 17 & 53.1 & 3 & 9.3 \\
\hline Mucositis & 5 & 15.6 & 0 & 0 \\
\hline Hand-foot syndrome & 11 & 34.3 & 1 & 3.1 \\
\hline Peripheral neuropathy & 26 & 81.2 & 5 & 15.6 \\
\hline Allergy & 3 & 9.3 & 1 & 3.1 \\
\hline
\end{tabular}

investigated the effects of oxaliplatin-based neoadjuvant CRT and adjuvant chemotherapy in Stage II/III rectal cancer [25]. Only the ADORE randomized phase II study showed that adjuvant FOLFOX improved disease-free survival compared with fluorouracil plus leucovorin in patients with locally advanced rectal cancer after preoperative CRT and total mesorectal excision [19]. The 3-year RFS and OS rates in the present trial were comparable to those of NSAS-CC and ACTS-RC trials which used an oral fluoropyrimidine alone as adjuvant chemotherapy. The reason for not showing superiority of adding oxaliplatin to fluoropyrimidine may involve differences in patient characteristics. In the present trial, the main tumor was located in the rectum with the lower margin below the peritoneal reflection and the proportion of patients with T4 tumors was $30 \%$. However, the NSAS-CC trial included patients with RS tumors, and only $10 \%$ of patients had T4 tumors in the ACTS-RC trial. To demonstrate the benefit of adding oxaliplatin to fluoropyrimidine, a study comparing oral fluoropyrimidine monotherapy with a combination therapy comprising oxaliplatin and oral fluoropyrimidine as adjuvant chemotherapy for rectal cancer will be needed.

Surgical treatment outcomes in our cohort were favorable. The $R 0$ resection rate was $97.5 \%$ and the rate of anastomotic leakage was $10 \%$. With regard to neoadjuvant therapy for locally advanced rectal cancer, a major issue is the increase in complications of curative surgery with preoperative therapy. According to a recent clinical trial, CRT increased perioperative complications such as anastomotic leakage and surgical site infection [24]. Moreover, the rate of anastomotic leakage in the present trial was lower than that reported in a phase II trial of perioperative
CapOX without radiotherapy for high-risk rectal cancer (CORONA I study) [26].

The MOSAIC, NSABPC-07, and NO16968 trials have demonstrated the superiority of adding oxaliplatin to fluoropyrimidine in the adjuvant setting for Stage III colon cancer, irrespective of how fluoropyrimidine is delivered (e.g., intravenous infusion, bolus, or oral) [9-11]. The present trial used an oral fluoropyrimidine (capecitabine), which is potentially more convenient for patients and oncologists compared with intravenous 5-FU-based regimens. Kopec et al. [27] reported that patients who received adjuvant therapy with oral fluoropyrimidine perceived it to be more convenient than intravenous 5-FU. We believe that CapOX reduces medical resource use (e.g., drug administration visits, central venous port placement, and removal) and places less of a time burden on patients compared with FOLFOX.

In terms of safety, the most frequent adverse events associated with adjuvant CapOX therapy were PSN and hematologic events, such as anemia and thrombocytopenia. Of Grade $\geq 3$ adverse events, frequencies for PSN, neutropenia, and thrombocytopenia were $15.6 \%, 9.3 \%$, and $12.5 \%$, respectively. These findings are consistent with those of the NO16968 trial [28]. PSN resulting from the use of oxaliplatin is a cumulative dose-related toxicity. In the present trial, $25 \%$ of patients had persistent Grade $1 / 2$ neurosensory toxicity at 12 months after the end of adjuvant treatment. Pachman et al. reported that about $30 \%$ of patients experienced moderate neuropathy as measured by the European Organisation for Research and Treatment of Cancer Quality of Life Questionnaire for patients with chemotherapyinduced peripheral neuropathy (EORTC-CIPN) and about $20 \%$ of patients experienced severe neuropathy at 18 months from adjuvant oxaliplatin therapy [29]. Thus, a shorter duration of adjuvant CapOX therapy would be beneficial so long as efficacy is not affected. In this regard, the ACHIEVE trial confirmed that a shorter duration of oxaliplatin-based adjuvant chemotherapy resulted in a significant reduction of PSN in a Japanese population with Stage III colon cancer [30].

This trial has a number of limitations worth noting. First, the single-arm design and relatively small sample size necessitate confirmation of our results in a larger cohort. Second, the numbers of cases with bulky tumors $>10 \mathrm{~cm}$, with T4, and that were lymph node-positive were fewer than those of the CORONA I study. It will be necessary to carefully consider curative resection with adjuvant chemotherapy alone for all Stage II/III rectal cancer cases and to consider treatment strategies including perioperative chemotherapy based on the state of tumor progression.

In conclusion, we found that CapOX as adjuvant chemotherapy for locally advanced rectal cancer is a feasible regimen. CapOX may serve as an important option for patients who have not undergone preoperative therapy. 
Acknowledgements The following physicians and research coordinators provided care to the patients in this study: $\mathrm{K}$. Taniguchi and $\mathrm{H}$. Yokoyama (H. Komaki); S. Takeda (H. Nagoya Medical Center); Y. Okada (H. Handa); and M. Aoki and H. Hattori (CCOG data center). The authors thank these individuals for their cooperation and support. The results of this study were presented in part at 56th Annual Meeting of Japan Society of Clinical Oncology, October 18-20, Yokohama, Japan.

\section{Compliance with ethical standards}

Conflict of interest The authors report no conflicts of interest.

Open Access This article is distributed under the terms of the Creative Commons Attribution 4.0 International License (http://creativeco mmons.org/licenses/by/4.0/), which permits unrestricted use, distribution, and reproduction in any medium, provided you give appropriate credit to the original author(s) and the source, provide a link to the Creative Commons license, and indicate if changes were made.

\section{References}

1. Siegel RL, Miller KD, Fedewa SA et al (2017) Colorectal cancer statistics, 2017. CA Cancer J Clin 67:177-193

2. Watanabe T, Muro K, Ajioka Y et al (2018) Japanese society for cancer of the colon and rectum (JSCCR) guidelines 2016 for the treatment of colorectal cancer. Int J Clin Oncol 23:1-34

3. Bosset JF, Collette L, Calais G et al (2006) Chemotherapy with preoperative radiotherapy in rectal cancer. $\mathrm{N}$ Engl $\mathrm{J}$ Med 355:1114-1123

4. Gerard JP, Conroy T, Bonnetain F et al (2006) Preoperative radiotherapy with or without concurrent fluorouracil and leucovorin in T3-4 rectal cancers: results of FFCD 9203. J Clin Oncol 24:4620-4625

5. Bujko K, Nowacki MP, Nasierowska-Guttmejer A et al (2006) Long-term results of a randomized trial comparing preoperative short-course radiotherapy with preoperative conventionally fractionated chemoradiation for rectal cancer. Br J Surg 93:1215-1223

6. Ngan SY, Burmeister B, Fisher RJ et al (2012) Randomized trial of short-course radiotherapy versus long-course chemoradiation comparing rates of local recurrence in patients with $\mathrm{T} 3$ rectal cancer: trans-tasman radiation oncology group trial 01.04. J Clin Oncol 30:3827-3833

7. Twelves C, Wong A, Nowacki MP et al (2005) Capecitabine as adjuvant treatment for stage III colon cancer. N Engl J Med 352:2696-2704

8. Lembersky BC, Wieand HS, Petrelli NJ et al (2006) Oral uracil and tegafur plus leucovorin compared with intravenous fluorouracil and leucovorin in stage II and III carcinoma of the colon: results from national surgical adjuvant breast and bowel project protocol C-06. J Clin Oncol 24:2059-2064

9. Andre T, Boni C, Navarro M et al (2009) Improved overall survival with oxaliplatin, fluorouracil, and leucovorin as adjuvant treatment in stage II or III colon cancer in the MOSAIC trial. J Clin Oncol 27:3109-3116

10. Haller DG, Tabernero J, Maroun J et al (2011) Capecitabine plus oxaliplatin compared with fluorouracil and folinic acid as adjuvant therapy for stage III colon cancer. J Clin Oncol 29:1465-1471

11. Yothers G, O'Connell MJ, Allegra CJ et al (2011) Oxaliplatin as adjuvant therapy for colon cancer: updated results of NSABP
C-07 trial, including survival and subset analyses. J Clin Oncol 29:3768-3774

12. Shimada Y, Hamaguchi T, Mizusawa J et al (2014) Randomised phase III trial of adjuvant chemotherapy with oral uracil and tegafur plus leucovorin versus intravenous fluorouracil and levofolinate in patients with stage III colorectal cancer who have undergone Japanese D2/D3 lymph node dissection: final results of JCOG0205. Eur J Cancer 50:2231-2240

13. Yoshida M, Ishiguro M, Ikejiri K et al (2014) S-1 as adjuvant chemotherapy for stage III colon cancer: a randomized phase III study (ACTS-CC trial). Ann Oncol 25:1743-1749

14. Hamaguchi T, Shirao K, Moriya Y et al (2011) Final results of randomized trials by the National Surgical Adjuvant Study of Colorectal Cancer (NSAS-CC). Cancer Chemother Pharmacol 67:587-596

15. Oki E, Murata A, Yoshida K et al (2016) A randomized phase III trial comparing S-1 versus UFT as adjuvant chemotherapy for stage II/III rectal cancer (JFMC35-C1: ACTS-RC). Ann Oncol 27:1266-1272

16. Glynne-Jones R, Wyrwicz L, Tiret E et al (2017) Rectal cancer: ESMO Clinical Practice Guidelines for diagnosis, treatment and follow-up. Ann Oncol 28:iv22-iv40

17. Benson AB 3rd, Venook AP, Al-Hawary MM et al (2018) Rectal cancer, version 2.2018 , NCCN clinical practice guidelines in oncology. J Natl Compr Canc Netw 16:874-901

18. Gerard JP, Azria D, Gourgou-Bourgade S et al (2012) Clinical outcome of the ACCORD 12/0405 PRODIGE 2 randomized trial in rectal cancer. J Clin Oncol 30:4558-4565

19. Hong YS, Nam BH, Kim KP et al (2014) Oxaliplatin, fluorouracil, and leucovorin versus fluorouracil and leucovorin as adjuvant chemotherapy for locally advanced rectal cancer after preoperative chemoradiotherapy (ADORE): an open-label, multicentre, phase 2, randomised controlled trial. Lancet Oncol 15:1245-1253

20. Allegra CJ, Yothers G, O'Connell MJ et al (2015) Neoadjuvant 5 -FU or capecitabine plus radiation with or without oxaliplatin in rectal cancer patients: a phase iii randomized clinical trial. J Natl Cancer Inst 107:djv248

21. Aschele C, Cionini L, Lonardi S et al (2011) Primary tumor response to preoperative chemoradiation with or without oxaliplatin in locally advanced rectal cancer: pathologic results of the STAR-01 randomized phase III trial. J Clin Oncol 29:2773-2780

22. Rodel C, Graeven U, Fietkau R et al (2015) Oxaliplatin added to fluorouracil-based preoperative chemoradiotherapy and postoperative chemotherapy of locally advanced rectal cancer (the German CAO/ARO/AIO-04 study): final results of the multicentre, openlabel, randomised, phase 3 trial. Lancet Oncol 16:979-989

23. Jiao D, Zhang R, Gong Z et al (2015) Fluorouracil-based preoperative chemoradiotherapy with or without oxaliplatin for stage II/III rectal cancer: a 3-year follow-up study. Chin J Cancer Res 27:588-596

24. Deng Y, Chi P, Lan P et al (2016) Modified FOLFOX6 with or without radiation versus fluorouracil and leucovorin with radiation in neoadjuvant treatment of locally advanced rectal cancer: initial results of the chinese fowarc multicenter, open-label, randomized three-arm phase III trial. J Clin Oncol 34:3300-3307

25. Fu XL, Fang Z, Shu LH et al (2017) Meta-analysis of oxaliplatinbased versus fluorouracil-based neoadjuvant chemoradiotherapy and adjuvant chemotherapy for locally advanced rectal cancer. Oncotarget 8:34340-34351

26. Kamiya T, Uehara K, Nakayama G et al (2016) Early results of multicenter phase II trial of perioperative oxaliplatin and capecitabine without radiotherapy for high-risk rectal cancer: CORONA I study. Eur J Surg Oncol 42:829-835

27. Kopec JA, Yothers G, Ganz PA et al (2007) Quality of life in operable colon cancer patients receiving oral compared with 
intravenous chemotherapy: results from national surgical adjuvant breast and bowel project trial C-06. J Clin Oncol 25:424-430

28. Schmoll HJ, Cartwright T, Tabernero J et al (2007) Phase III trial of capecitabine plus oxaliplatin as adjuvant therapy for stage III colon cancer: a planned safety analysis in 1,864 patients. J Clin Oncol 25:102-109

29. Pachman DR, Qin R, Seisler DK et al (2015) Clinical course of oxaliplatin-induced neuropathy: results from the randomized phase III trial N08CB (Alliance). J Clin Oncol 33:3416-3422

30. Kotaka M, Yamanaka T, Yoshino T et al (2018) Safety data from the phase III Japanese ACHIEVE trial: part of an international, prospective, planned pooled analysis of six phase III trials comparing 3 versus 6 months of oxaliplatin-based adjuvant chemotherapy for stage III colon cancer. ESMO Open 3:e000354

Publisher's Note Springer Nature remains neutral with regard to jurisdictional claims in published maps and institutional affiliations. 